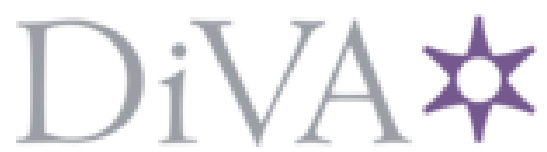

http://www.diva-portal.org

This is the published version of a paper published in Clinical Physiology and Functional Imaging.

Citation for the original published paper (version of record):

Ahlander, B-M., Maret, E., Brudin, L., Starck, S-A., Engvall, J. (2017)

An echo-planar imaging sequence is superior to a steady-state free precession sequence for visual as well as quantitative assessment of cardiac magnetic resonance stress perfusion

Clinical Physiology and Functional Imaging, 37(1): 52-61

https://doi.org/10.1111/cpf.12267

Access to the published version may require subscription.

N.B. When citing this work, cite the original published paper.

Permanent link to this version:

http://urn.kb.se/resolve?urn=urn:nbn:se:oru:diva-72073 


\title{
An echo-planar imaging sequence is superior to a steady-state free precession sequence for visual as well as quantitative assessment of cardiac magnetic resonance stress perfusion
}

\author{
Britt-Marie Ahlander ${ }^{1}$, Eva Maret ${ }^{1,2}$, Lars Brudin ${ }^{3}$, Sven-Ake Starck ${ }^{4,5}$ and Jan Engvall ${ }^{6,7,8}$ \\ ${ }^{1}$ Department of Radiology, Ryhov County Hospital, Jonkoping, ${ }^{2}$ Department of Clinical Physiology, Karolinska University Hospital, Stockholm, ${ }^{3}$ Department of \\ Clinical Physiology, Kalmar County Hospital, Kalmar, ${ }^{4}$ Department of Natural Science and Biomedicine, School of Health Sciences, Jonkoping University, \\ ${ }^{5}$ Department of Oncology, Hospital Physics, Ryhov County Hospital, Jonkoping, ${ }^{6}$ Department of Medical and Health Sciences, Linkoping University, ${ }^{7}$ Department \\ of Clinical Physiology, County Council of Ostergotland, and ${ }^{8}$ Center of Medical Image Science and Visualisation, Linkoping University, Linkoping Sweden
}

\section{Summary}

\section{Correspondence}

Jan Engvall, Center of Medical Image Science and

Visualisation, Linkoping University, SE-581 83

Linkoping, Sweden.

E-mail: jan.engvall@regionostergotland.se

\section{Accepted for publication}

Received 7 November 2014;

accepted 8 May 2015

\section{Key words}

cardiac imaging techniques; coronary heart disease; Magnetic Resonance Imaging; nuclear medicine; perfusion
Background To assess myocardial perfusion, steady-state free precession cardiac magnetic resonance (SSFP, CMR) was compared with gradient-echo-echo-planar imaging (GRE-EPI) using myocardial perfusion scintigraphy (MPS) as reference.

Methods Cardiac magnetic resonance perfusion was recorded in 30 patients with SSFP and in another 30 patients with GRE-EPI. Timing and extent of inflow delay to the myocardium was visually assessed. Signal-to-noise (SNR) and contrastto-noise (CNR) ratios were calculated. Myocardial scar was visualized with a phase-sensitive inversion recovery sequence (PSIR). All scar positive segments were considered pathologic. In MPS, stress and rest images were used as in clinical reporting. The CMR contrast wash-in slope was calculated and compared with the stress score from the MPS examination. CMR scar, CMR perfusion and MPS were assessed separately by one expert for each method who was blinded to other aspects of the study.

Results Visual assessment of CMR had a sensitivity for the detection of an abnormal MPS at 78\% (SSFP) versus $91 \%$ (GRE-EPI) and a specificity of 58\% (SSFP) versus 84\% (GRE-EPI). Kappa statistics for SSFP and MPS was $0 \cdot 29$, for GRE-EPI and MPS 0.72. The ANOVA of CMR perfusion slopes for all segments versus MPS score (four levels based on MPS) had correlation $r=0.64$ (SSFP) and $r=0.96$ (GREEPI). SNR was for normal segments $35.63 \pm 11.80$ (SSFP) and $17.98 \pm 8.31$ (GRE-EPI), while CNR was $28.79 \pm 10.43$ (SSFP) and $13.06 \pm 7.61$ (GRE-EPI). Conclusion GRE-EPI displayed higher agreement with the MPS results than SSFP despite significantly lower signal intensity, SNR and CNR.

\section{Introduction}

Myocardial ischaemia can be detected by the difference in myocardial signal intensity on cardiac magnetic resonance images recorded after stress and at rest. Normally, the extraction of oxygen in the myocardium is high, and an increase in myocardial oxygen demand requires an increase in coronary blood flow. In coronary arteries with a normal endothelial function and normal cross-sectional area, coronary blood flow may increase four times the resting level during vasodilation or dynamic exercise. This increase in flow is reported as coro- nary flow reserve (CFR) (Gould et al., 1990). The coronary vasculature in the perfusion area supplied by a stenotic vessel is already maximally dilated and displays a reduced response to the injection of adenosine in comparison with other myocardial segments. This mechanism is utilized for imaging differences between stress and rest perfusion with CMR, and with MPS using single photon emission computed tomography (SPECT) (Fleischmann et al., 2004; Gibbons et al., 2006), Fig. 1.

Myocardial perfusion scintigraphy is the pre-eminent clinical method for the evaluation of myocardial perfusion and 
uses exercise-induced flow reduction or the redistribution of flow from pharmacological vasodilation to define pathologic segments from those considered normal. The method has a reasonable diagnostic accuracy but requires the administration of radionuclide tracers (Zhang et al., 1998; Fleischmann et al., 2004; Gibbons et al., 2006; Marcassa et al., 2008) that will cause some radiation exposure to the patient.

Cardiac magnetic resonance imaging has emerged as an important method for the evaluation of coronary artery disease (CAD). It has a high spatial resolution, good signal-tonoise (SNR) and contrast-to-noise ratios (CNR) and requires neither X-rays nor radiotracers (Constantine et al., 2004; Nandalur et al., 2007; Greenwood et al., 2011). Late gadolinium enhancement (LGE) imaging is the gold standard (Sakuma, 2007) for visualizing myocardial scar. After intravenous injection, gadolinium accumulates in the extracellular space in fibrotic non-viable myocardium and washes out slowly, enhancing the magnetic resonance (MR) signal of scar tissue by shortening the T1 relaxation time (Finn et al., 2006; Sakuma, 2007). In first pass perfusion imaging, a contrast bolus traverses the pulmonary circulation and the left ventricle to produce an increase in MR signal in the left ventricular wall. Gadolinium contrast material in highly perfused myocardium appears bright, while hypoperfused areas have less (darker) signal (Barkhausen et al., 2004; Gerber et al., 2008; Kim et al., 2009). This difference in signal intensity (SI) can be evaluated visually, semiquantitatively or quantitatively (Gerber et al., 2008; Jerosch-Herold, 2010), Fig. 2. Visual assessment is fast but requires experienced investigators that can differentiate true perfusion reduction from 'dark rim' artefact (Di Bella et al., 2005), Fig. 3.
Objective measurements to detect segmental ischaemia are based on, for example, stress-rest differences in the slope of the signal intensity curve of the myocardium or a reduction in subendocardial compared to epicardial blood flow using Fermi deconvolution to determine absolute blood flow (Mordini et al., 2014).

Magnetic resonance sequences used for perfusion need to have a high temporal and spatial resolution. Three short axis slices with six segments in each slice (Cerqueira et al., 2002) cover all three levels of the left ventricle (excluding the apical cap). Strong T1 weighting is required for visualization of differences in contrast density (Kellman and Arai, 2007). Perfusion sequences have been designed based on gradient-echoecho-planar imaging, GRE-EPI, or steady-state free precession, SSFP (Kellman and Arai, 2007; Gerber et al., 2008). Based on the properties of SSFP, with a high SNR and CNR, we hypothesized that an SSFP sequence could have advantages in cardiac perfusion compared with the GRE-EPI sequence (Wang et al., 2005; Gebker et al., 2007; Merkle et al., 2007).

The aim of this study was to compare first pass stress myocardial perfusion CMR, obtained with two different sequences, with each other using the result from the MPS stress study as reference. Angiography was not part of the study and was later available only for seven of the 60 patients.

\section{Methods}

\section{Patients}

Sixty patients (mean age 62 years, range 37-80, 23 women), Table 1, referred for MPS for myocardial ischaemia were

Figure 1 SPECT ( $1 \mathrm{a}$ and $1 \mathrm{~b}$ ) and CMR GREEPI ( $2 a$ and $2 b)$ images of reversible myocardial ischaemia. Stress is 'a' and rest ' $b$ '.
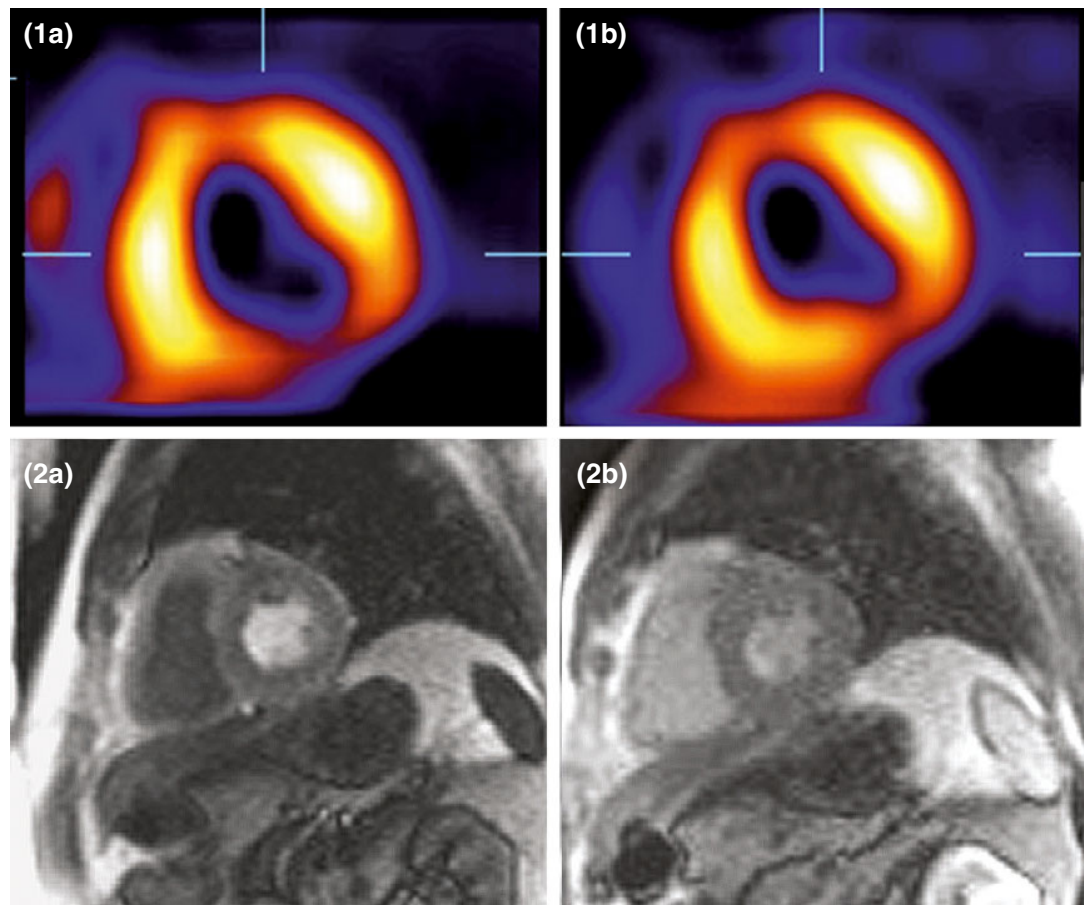

(C) 2015 Scandinavian Society of Clinical Physiology and Nuclear Medicine. Published by John Wiley \& Sons Ltd 37, 1, 52-61 

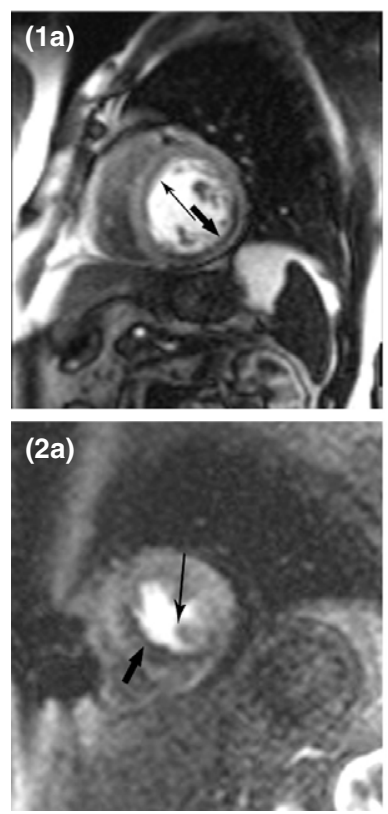
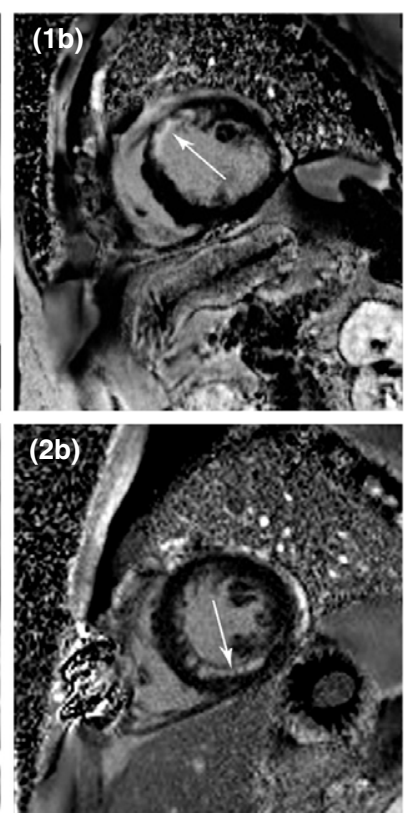
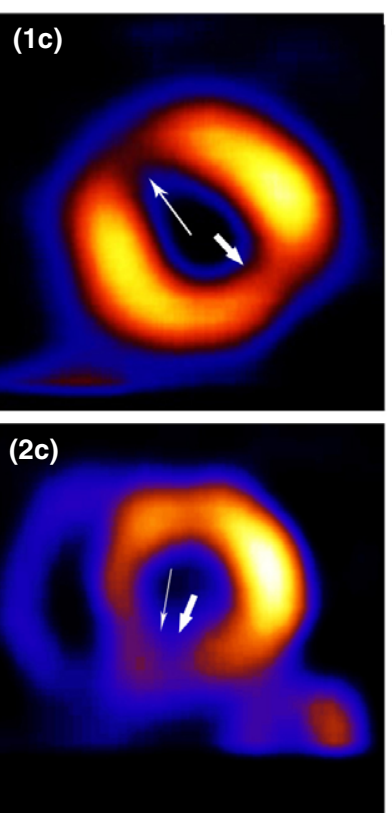

(1d)

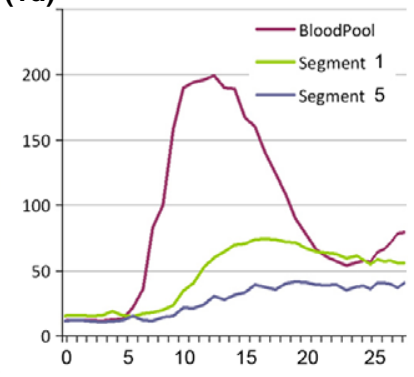

(2d)

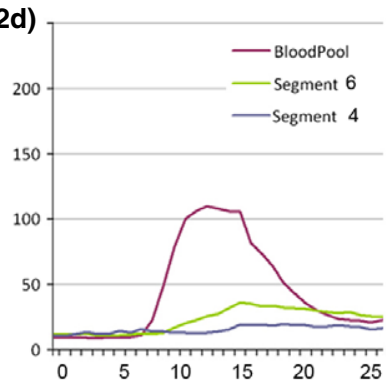

Figure 2 Composite image of two patient studies with ischaemia and myocardial scar, SSFP in 1 and GRE-EPI in 2. Perfusion with SSFP sequence (1a), anteroseptal scar visualized with LGE sequence (1b), corresponding MPS image (1c) and contrast wash-in curves (1d) for the bloodpool (red), a pathologic segment (blue) and a normal segment (green). Perfusion with GRE-EPI sequence (2a), inferoseptal scar (2b), MPS image (2c) and wash-in curves (2d). Annotation as in 1d. Segment numbers according to SCMR. Scar is indicated by thin arrows and ischaemia by thick arrows. In wash-in curves, MRI contrast signal intensity is depicted on the $y$-axis and time (s) on the $x$-axis.
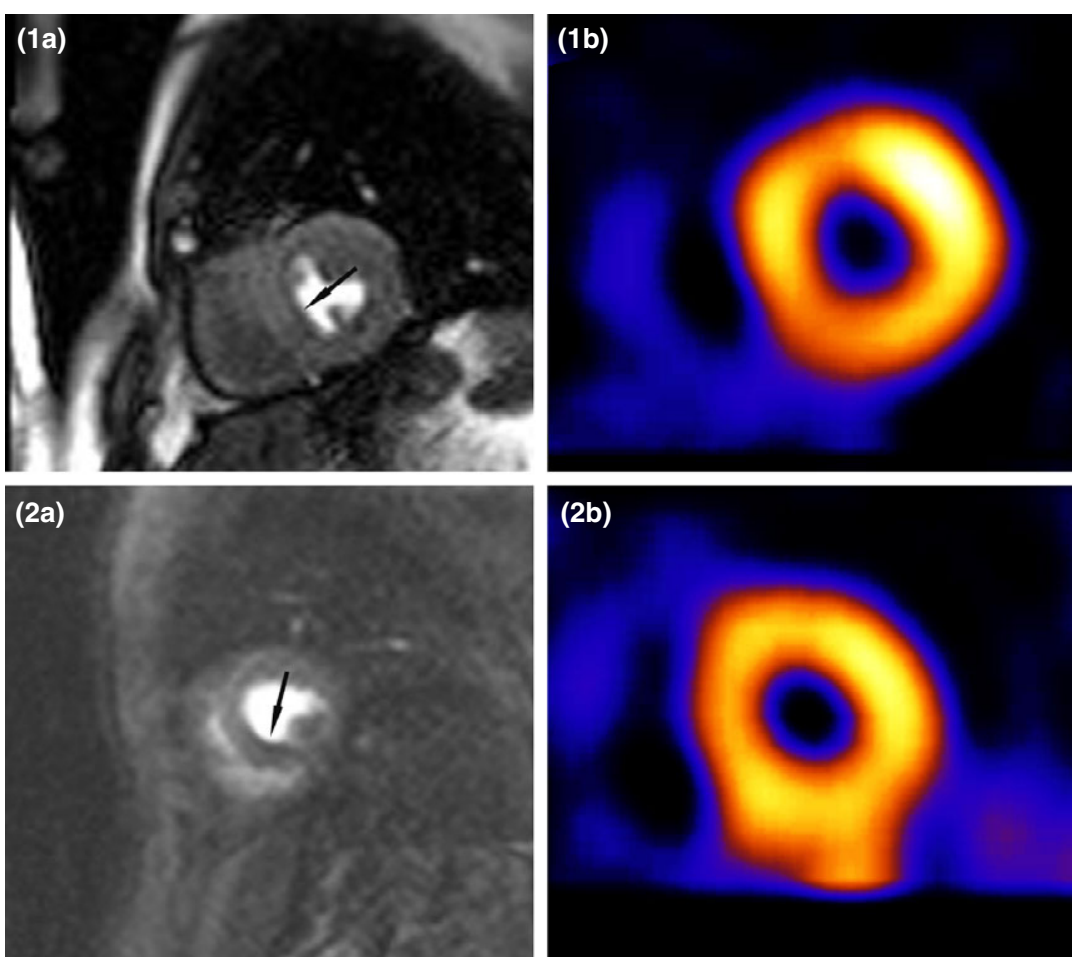

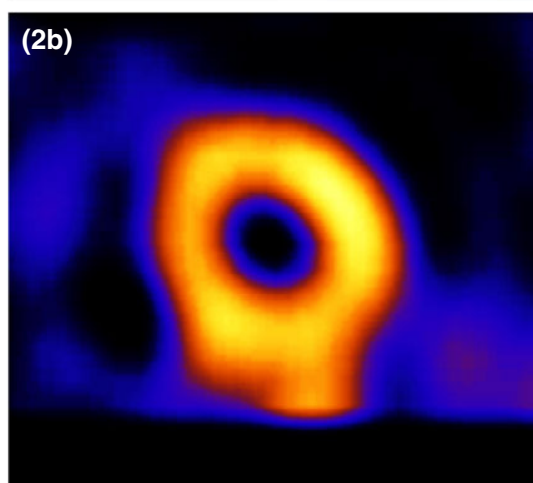

Figure 3 Dark rim artefact at arrows on CMR perfusion images using SSFP (1a) and GRE-EPI (2a) sequences. Corresponding normal MPS images (1b and 2b). Slight extracardiac bowel isotope uptake signal in $2 \mathrm{~b}$. enrolled in the study between April 2008 and June 2011. In the initial phase from April 2008 to April 2009 30, patients were investigated with an SSFP sequence, while in the period between November 2009 and June 2011 30, patients were investigated with the GRE-EPI sequence. Exclusion criteria were as follows: contraindications for magnetic resonance imaging, to the use of adenosine vasodilator or gadolinium contrast, inability to communicate or unwillingness to participate.

The study was approved by the regional ethical review board in Linköping, Sweden, and adhered to Good Clinical 
Table 1 Clinical characteristics for the 30 patients in each MR perfusion group; steady-state free precession (SSFP) and gradient-echoecho-planar imaging (GRE-EPI).

\begin{tabular}{lrcc}
\hline Characteristics & $\begin{array}{c}\text { SSFP } \\
(\boldsymbol{n}=\mathbf{3 0})\end{array}$ & $\begin{array}{c}\text { GRE-EPI } \\
(\boldsymbol{n}=\mathbf{3 0})\end{array}$ & $\boldsymbol{P}^{\text {-value }} \mathbf{v a}^{\mathbf{2}}$ \\
\hline Gender n (\%) & & & \\
$\quad$ Male & $17(57)$ & $20(67)$ & \\
$\quad$ Female & $13(43)$ & $10(33)$ & $0 \cdot 595$ \\
Age year; mean (SD) & $60(9 \cdot 3)$ & $64(10 \cdot 3)$ & $0 \cdot 051$ \\
BMI kg m ${ }^{-2}$; mean (SD) & $26(4 \cdot 2)$ & $27(3 \cdot 6)$ & $0 \cdot 273$ \\
Diabetes n (\%) & $3(10)$ & $7(23)$ & $0 \cdot 490$ \\
Hypertension n (\%) & $14(47)$ & $21(70)$ & $0 \cdot 115$ \\
Smoker n (\%) & $5(17)$ & $4(13)$ & $0 \cdot 735$ \\
Ischaemic heart disease n (\%) & $26(87)$ & $29(97)$ & $0 \cdot 353$ \\
Angina pectoris & $25(83)$ & $29(97)$ & $0 \cdot 194$ \\
Infarction & $8(27)$ & $15(50)$ & $0 \cdot 110$ \\
PCI & $9(30)$ & $10(33)$ & $>0 \cdot 9$ \\
CBAG & $4(13)$ & $3(10)$ & $>0 \cdot 9$ \\
Peripheral vascular disease n (\%) & $7(23)$ & $2(7)$ & $0 \cdot 145$ \\
Medication n (\%) & & & \\
Betablocker & $17(57)$ & $21(70)$ & $0 \cdot 422$ \\
Calcium & $7(23)$ & $11(37)$ & $0 \cdot 398$ \\
Statin & $19(63)$ & $17(57)$ & $0 \cdot 792$ \\
ACI-I & $10(33)$ & $18(60)$ & $0 \cdot 069$ \\
Blood pressure; mean (SD) & & & \\
Systolic & $137(21 \cdot 9)$ & $140(19 \cdot 2)$ & $0 \cdot 730$ \\
Diastolic & $78(12 \cdot 1)$ & $77(9 \cdot 6)$ & $0 \cdot 476$ \\
Follow-up n (\%) & & & \\
MI & $4(13)$ & $3(10)$ & $>0 \cdot 9$ \\
PCI & $7(23)$ & $4(13)$ & $0 \cdot 506$ \\
Angiography & $6(20)$ & $3(10)$ & $0 \cdot 471$ \\
CABG & $0(0)$ & $1(3)$ & $>0 \cdot 9$ \\
\hline
\end{tabular}

${ }^{a}$ Differences between groups analysed using Mann-Whitney non-parametric U-test for continuous parameters and Fisher's exact test for categorical (frequencies).

${ }^{\mathrm{b}}$ Follow-up is performed April 2015, 7-4 years after CMR.

Practice as set forth in the declaration of Helsinki. Written informed consent was obtained from all patients after the nature of the procedures had been fully explained.

\section{Cardiac Magnetic Resonance Imaging}

All examinations were performed on an 1.5-T MRI scanner (Magnetom Avanto; Siemens Healthcare, Erlangen, Germany) with a 6-element phased array body matrix coil combined with six elements in the spine coil, altogether 12 elements. All images were acquired in supine position and in end diastole during breathhold. Gadopentetate dimeglumine contrast $0.5 \mathrm{mmol} \mathrm{ml}^{-1}$ (Magnevist; Bayer Schering Pharma, Berlin, Germany) and adenosine $5 \mathrm{mg} \mathrm{ml}^{-1}$ (Item Development AB, Stocksund, Sweden) was used for stress imaging. Electrocardiogram (ECG) and heart rate were monitored during the entire examination, while blood pressure was checked only before the patient entered the scanner room. After scout images, the scanning table was moved outside the tunnel to facilitate control of side effects and the adenosine infusion was started $(140 \mu \mathrm{g}$ min per $\mathrm{kg}$ body weight). Patients had been instructed to withhold caffeine for $24 \mathrm{~h}$ (Carlsson et al., 2014). After three minutes, 99m Tc tetrofosmin (for the MPS study) was given followed by a sodium chloride chaser and the table moved to the scanning position. When the correct scanning position was reached, $8 \mathrm{ml}$ gadolinium contrast was infused using a power injector (Medrad Inc, Indianola, PA, USA) at a rate of $4 \mathrm{ml} \mathrm{s}^{-1}$ during breathhold, while the vasodilator infusion was still running.

For perfusion analysis, three 8 -mm-thick short axis images were equally spaced along the left ventricular long axis in the middle position of the basal, mid- and apical segments. The perfusion sequences were ECG-gated with non-selective saturation recovery preparation pulses. The sequence parameters for SSFP were TR/TE/TI/FA $172 \cdot 7 / 1 \cdot 11 / 100 \mathrm{~ms} / 50^{\circ}$, raw data matrix $60 \times 160$, field of view (FOV) $250 \times 380 \mathrm{~mm}^{2}$, bandwidth (BW) $1359 \mathrm{~Hz}$ per pixel and voxel spatial resolution $3.2 \times 2.4 \times 8 \mathrm{~mm}^{3}$. Depending on patient size, FOV varied from $225 \times 300$ to $330 \times 440 \mathrm{~mm}^{2}$ and pixel size from $2.5 \times 1.9$ to $3.7 \times 2.8 \mathrm{~mm}^{2}$. For GRE-EPI, the parameters were TR/TE/TI/FA $146 \cdot 95 / 1 \cdot 26 / 115 \mathrm{~ms} / 20^{\circ}$, raw data matrix of $102 \times 128$, FOV $281 \times 360 \mathrm{~mm}^{2}$, BW1628 $\mathrm{Hz} /$ pixel and voxel spatial resolution $3.5 \times 2.8 \times 8 \mathrm{~mm}^{3}$. In this sequence, FOV varied from $273 \times 350$ to $351 \times 450 \mathrm{~mm}^{2}$ resulting in a pixel size of $2.7 \times 3.3-$ $3.5 \times 4.4 \mathrm{~mm}^{2}$. Parallel imaging, GeneRalized Autocalibrating Partially Parallel Acquisition (GRAPPA) (Griswold et al., 2002), with an acceleration factor 2, was used in the phase-encoding direction. First pass perfusion images at rest were acquired $10 \mathrm{~min}$ after the stress study using a second bolus of $8 \mathrm{ml}$ gadolinium contrast and identical scanner settings. The perfusion contrast dose corresponded to $0.05 \mathrm{mmol} \mathrm{kg}^{-1}$ for an $80 \mathrm{~kg}$ individual which is a dose recommended by SCMR (Kramer et al., 2008). For scar imaging, a third contrast injection was given aiming at a total contrast dose of $0.2 \mathrm{mmol} \mathrm{kg}^{-1}$. However, for practical reasons, a maximal dose of $30 \mathrm{ml}$ was used. 28 of the 60 patients weighed more than $75 \mathrm{~kg}$ and were subject to this limitation in dosage. Cine images for ventricular function and LGE images for scar evaluation were acquired before the patient moved to the nuclear department where MPS was performed about $60 \mathrm{~min}$ after the injection of the radiotracer.

\section{Image analysis of first pass perfusion CMR}

Image analysis was performed after the conclusion of the study, on the entire batch of study patients. First pass perfusion CMR during stress and at rest was qualitatively evaluated using visual assessment of the presence of delayed wash-in of contrast. Ischaemia was deemed likely if the delay was not being present in the rest images, and artefact was deemed likely if the reduction was short-lived (four beats or less) and affected a shallow depth of the LV wall (Hundley et al., 2009). The level of diagnostic confidence was given on a four-point scale: (i) normal with high confidence, (ii) normal 
with low confidence, (iii) pathologic with low confidence and (iv) pathologic with a high confidence (Schwitter et al., 2008). A difference of two steps between stress and rest was required to determine that a specific segment was ischaemic. In the semiquantitative evaluation, the slope of the signal increase in the myocardium was measured on a work station using 'Argus Dynamic Signal ${ }^{\mathrm{TM}}$ ' (Siemens Healthcare). Each slice was automatically divided into six segments, creating 18 $(3 \times 6)$ segments in every patient excluding the apical cap. Thus, the evaluation differed slightly from the 17-segment model recommended by the American Heart Association (Taylor et al., 2010). The epicardial and endocardial borders were manually segmented excluding the high signal of blood in the cavity and the epicardial fat surrounding the left ventricle. Segmentation was repeated for each time step and the signal intensity curve recorded for each segment. The slope of the inflow signal was calculated between the foot and the peak of the curve; thus, the derivative of the slope was not used.

Reproducibility for the slope measurement was based on two independent observers evaluating five patients each for both sequences. Qualitative evaluation of first pass perfusion CMR was performed by an experienced reader of cardiac MRI (>10 years) who was blinded to the MPS result. To optimize specificity and sensitivity, LGE images were used to identify areas of scar. An LGE positive segment was always considered pathological (Kramer, 2006).

For each patient, SNR and CNR were calculated in the anterior segment of the basal left ventricle when healthy and in all ischaemic segments, before and after the infusion of gadolinium contrast, for both perfusion sequences. Baseline SI was chosen as the value before the start of the infusion and peak SI as the highest value during contrast infusion. Noise was defined as the standard deviation of the signal in air outside the patient. SNR was calculated by dividing SI with noise. CNR for the contrast enhanced myocardium during perfusion compared with the myocardium before perfusion was calculated as $\left(\mathrm{SI}_{\text {Myocard perfusion }}-\mathrm{SI}_{\text {Myocard baseline) }} /\right.$ noise.

\section{Myocardial perfusion scintigraphy}

The perfusion images from the stress study were used for evaluation. During adenosine stress in the MR scanner, $5.7 \mathrm{MBq}{ }^{99 \mathrm{~m}}$ Tc tetrofosmin per $\mathrm{kg}$ bodyweight was given i.v. (max $570 \mathrm{MBq}$ ) (Myoview ${ }^{\mathrm{TM}}$, GE-Healthcare Medi-Physics, Inc, Arlington Heights, IL, USA). MPS imaging commenced $60 \mathrm{~min}$ after injection of the radiotracer. A dual-detector gamma camera (E. CAM; Siemens Medical Systems Inc, Hoffman Estates, IL, USA) equipped with a high resolution collimator was used. Thirty-two views were acquired in steps of $2 \cdot 8$ degrees per detector, and the acquisition time/angle was 30 s. A $19 \%$ window was 'asymmetrically placed' (129$155 \mathrm{keV}$ ) on the $140 \mathrm{keV}$ peak. A $64 \times 64$ word matrix with a pixel size of $6.6 \mathrm{~mm}$ was used. The studies were acquired simultaneously in both non-gated and ECG-gated mode.

\section{Image analysis of myocardial perfusion scintigraphy}

The non-gated acquisition files were reconstructed using filtered back projection, prefiltered with a Butterworth filter (cut-off $0.8 \mathrm{~cm}^{-1}$, order 10), (Hermes Medical Solutions, Stockholm, Sweden). The images were realigned into short axis slices in two phases, transverse rotation followed by oblique rotation. In case of interfering bowel uptake, acquisition was repeated after intake of fluids. Attenuation correction or prone imaging was not performed.

The images were analysed with QGS-QPS Quantitative Perfusion SPECT (Cedars-Sinai Medical Center, Los Angeles, CA, USA). The stress perfusion polar map was divided in 20 segments, six in each basal, mid- and apical area and two segments in the apex. The apical segments were not used as the CMR method could not visualize this area. Stress scores were given according to reference standards incorporated in the QPS software (based on segmental differences in signal intensity as seen in a healthy reference population). Using these scores, segments were reported as (0) normal, (1) probably normal, (2) probably diseased and (3 and 4) definitely diseased. For the visual comparison, both stress and rest images were used and assessed by an experienced nuclear physician (>10 years of experience) who was blinded to the results of the evaluation of MR perfusion.

\section{Statistics}

Descriptive statistics was used for both qualitative and quantitative evaluation of the agreement between the two CMR sequences in relation to MPS. For visual assessment, on a patient level, cross-tabulation of the binary data (normal-ischaemic/scar CMR and normal reversible/not reversible MPS) was performed. For proportion of agreement between MPS and SSFP and GRE-EPI respectively, kappa was calculated (Landis and Koch 1977). Sensitivity and specificity for detecting patients with ischaemic heart disease, with MPS as reference, was calculated for both sequences. Intraclass correlation with 95\% confidence interval was used for the calculation of interobserver variability. According to the Kolmogorov-Smirnov and Shapiro-Wilk tests, the distribution of CMR slope as well as MPS scores was skewed which necessitated values to be normalized to the peak value in each individual giving symmetrical and well-normalized distributions. The contrast wash-in slope was calculated for each segment and compared with the stress score from the MPS examination, using ANOVA. Region (basal, middle and apical), total number of segments, stress score and patient were used as input parameters.

Student's t-test for independent samples was used for comparison between the sequences regarding SI, SNR and CNR. A P-value $\leq 0.05$ was considered significant. All analyses were performed using Statistica version 10 (Statsoft Inc. Tulsa, OK, USA). 


\section{Results}

\section{Patients}

Sixty seven patients were initially enrolled of which seven were excluded for the following reasons: lack of two venous access lines (1), claustrophobia (4), scanner problem (1), arrhythmia (1). Patient mean age was $62 \pm 10$ years, and 23 were women. Patients in the group examined with GRE-EPI were slightly older, had a higher proportion of diabetes, hypertension and previous infarction, Table 1. Peripheral vascular disease was more frequent in the group examined with the SSFP sequence.

\section{Effects of adenosine}

The administration of adenosine increased heart rate from $64 \pm 12$ to $89 \pm 14$ (SSFP) and from $63 \pm 10$ to $83 \pm 16$ (GRE-EPI). All patients had an increase in heart rate exceeding 10 beats $\mathrm{min}^{-1}$. An increase in heart rate of $10 \%$ is frequently seen as the lower limit for an adequate hemodynamic response to vasodilation.

\section{Late gadolinium enhancement}

Myocardial scar was present in 10 pts from each perfusion sequence. For SSFP, the mean scar size/left ventricular mass (LVM) was $7 \cdot 40 \% \pm 9 \cdot 03$ and for GRE-EPI $7 \cdot 10 \% \pm 5 \cdot 51$. Scar size did not differ between the two sequences, $\mathrm{P}=0.92$ (Mann-Whitney U-test).

\section{Myocardial volumes derived from CMR and MPS}

The end-diastolic volume of the left ventricle (LVEDV) derived from CMR was $145 \pm 34 \mathrm{ml}$ (SSFP) and $161 \pm 43 \mathrm{ml}$ (GRE-EPI). Left ventricle ejection fraction (EF) was $58 \pm 11 \% \quad$ (SSFP) and $61 \pm 11 \% \quad$ (GRE-EPI). Using MPS, LVEDV was $107 \pm 43 \mathrm{ml}$ (SSFP) and $115 \pm 51 \mathrm{ml}$ (GRE-EPI). Ejection fraction was $54 \pm 11 \%$ (SSFP) and $56 \pm 11 \%$ (GRE-EPI), Table 2. Ejection fraction and volume measurements were not statistically different between the two MR cohorts $(P>0 \cdot 05)$.

Table 2 End-diastolic volume and ejection fraction measured with CMR and MPS for the two sequence groups. Measurements are mean value $\pm \mathrm{SD}$.

\begin{tabular}{lrrr}
\hline & \multicolumn{1}{c}{ SSFP $^{\mathbf{a}}$} & GRE-EPI $^{\mathbf{b}}$ & $\boldsymbol{P}^{\text {-value }}$ \\
\hline SPECT LVEDV ml & $107 \pm 43$ & $115 \pm 51$ & $0 \cdot 49$ \\
SPECT EF \% & $54 \pm 11$ & $56 \pm 11$ & $0 \cdot 37$ \\
CMR LVEDV ml & $145 \pm 34$ & $161 \pm 43$ & $0 \cdot 11$ \\
CMR EF\% & $58 \pm 11$ & $61 \pm 11$ & $0 \cdot 21$ \\
\hline
\end{tabular}

${ }^{\mathrm{a}}$ Steady-state free precession.

${ }^{\mathrm{b}}$ Gradient-echo-echo-planar imaging.

\section{SNR and CNR for the two CMR sequences}

At peak gadolinium, signal intensity for normal segments was $67 \cdot 72 \pm 6.40$ for SSFP versus $39.43 \pm 16 \cdot 86$ for GRE-EPI. SNR and CNR were as expected higher for SSFP than for the GRE-EPI sequence. SNR was for normal segments $35.63 \pm 11.80$ (SSFP) and $17.98 \pm 8.31$ (GRE-EPI), while CNR was $28.79 \pm 10.43$ (SSFP) and $13.06 \pm 7.61$ (GREEPI). But, segments with definite ischaemia (rated 3 or 4 ) had SNR $32 \cdot 31 \pm 13 \cdot 31$ (SSFP) versus SNR 15.71 $\pm 7 \cdot 87$ (GREEPI), while CNR was $25 \cdot 18 \pm 12.48 \quad$ (SSFP) and $10 \cdot 41 \pm 7 \cdot 66$ (GRE-EPI). In a comparison of SNR and CNR between the two sequence groups, these pairwise differences were all statistically significant, but the difference in SNR and CNR between normal and ischaemic segments was non-significant for SSFP as well as for GRE-EPI, Table 3.

\section{Visual assessment of MPS and CMR}

Visual assessment of MPS showed signs of coronary artery disease in 20 pats (ischaemia or scar) of which 13 demonstrated reversible ischaemia. The corresponding numbers for the two MRI sequences altogether were 26 and 21, Table 4. Using MPS as reference, the sensitivity for the detection of an abnormal CMR was 78\% (SSFP) versus 91\% (GRE-EPI), while specificity was 58\% (SSFP) and 84\% (GRE-EPI). Kappa statistics for the agreement between GRE-EPI and MPS was 0.72 and for SSFP 0.29 (Landis and Koch, 1977), but this was not statistically significant, $P=0 \cdot 07$, Fischer's exact test.

\section{Quantitative segmental CMR and MPS}

The slope of myocardial CMR contrast wash-in during vasodilation was compared with MPS summed stress scores, Fig. 4. For all three levels of the left ventricle, basal, mid- and apex segments with a high MPS stress score had a lower rise in the CMR slope than segments with low MPS stress score. The

Table 3 SI, SNR and CNR calculated for normal and ischaemic segments, measured in regions where both ischaemic and normal segments were found. $\mathrm{CE}=$ contrast enhancement. Measurements are mean value $\pm \mathrm{SD}$. Whereas all comparisons between sequences were statistically significant, the difference between normal and ischaemic segments in SNR and CNR was not statistically significant for neither SSFP nor GRE-EPI. Measurements are mean value \pm SD.

\begin{tabular}{|c|c|c|c|}
\hline & SSFP $^{a}$ & GRE-EPI ${ }^{b}$ & $P$-value \\
\hline SI normal CE & $67 \cdot 72 \pm 6 \cdot 40$ & $39.43 \pm 16.86$ & 0.007 \\
\hline SI ischaemic CE & $62 \cdot 10 \pm 18 \cdot 35$ & $34.72 \pm 16 \cdot 81$ & 0.030 \\
\hline SNR normal & $35.63 \pm 11.80$ & $17 \cdot 98 \pm 8 \cdot 31$ & $0 \cdot 017$ \\
\hline SNR ischaemic & $32 \cdot 31 \pm 13 \cdot 31$ & $15 \cdot 71 \pm 7 \cdot 87$ & 0.030 \\
\hline CNR normal & $28.79 \pm 10.43$ & $13 \cdot 06 \pm 7,61$ & $0 \cdot 018$ \\
\hline CNR ischaemic & $25 \cdot 18 \pm 12 \cdot 48$ & $10 \cdot 41 \pm 7 \cdot 66$ & 0.039 \\
\hline
\end{tabular}

a Steady-state free precession.

${ }^{\mathrm{b}}$ Gradient-echo-echo-planar imaging. 
Table 4 Cross-tabulation of the visual assessment of CMR and MPS. MPS: normal or reversible/irreversible reduction of perfusion. CMR: normal, ischaemia or scar.

\begin{tabular}{|c|c|c|c|}
\hline \multirow[b]{2}{*}{$C M R^{a}$} & \multicolumn{3}{|c|}{ MPS $^{b}$} \\
\hline & Normal & $\begin{array}{l}\text { Reversible/ } \\
\text { irreversible }\end{array}$ & $\mathrm{Kappa}^{\mathrm{c}}$ \\
\hline \multicolumn{4}{|l|}{ SSFP $(\%)$} \\
\hline Normal & $12(57)$ & $9(43)$ & $0 \cdot 286$ \\
\hline Ischaemic/scar & $2(22)$ & $7(78)$ & \\
\hline \multicolumn{4}{|l|}{ GRE-EPI (\%) } \\
\hline Normal & $16(84)$ & $3(16)$ & 0.724 \\
\hline Ischaemic/scar & $1(9)$ & $10(91)$ & \\
\hline \multicolumn{4}{|l|}{ Total (\%) } \\
\hline Normal & $28(70)$ & $12(30)$ & $0 \cdot 494$ \\
\hline Ischaemic/scar & $3(15)$ & $17(85)$ & \\
\hline
\end{tabular}

${ }^{a}$ Cardiac magnetic resonance imaging.

${ }^{\mathrm{b}}$ Myocardial perfusion scintigraphy.

${ }^{\mathrm{c}} 0-0 \cdot 2$ : Poor agreement, $>0 \cdot 2-0 \cdot 4$, fair, $>0 \cdot 4-0.6$ moderate, $>0 \cdot 6-0 \cdot 8$ substantial and $>0 \cdot 8$ almost perfect agreement. $P=0 \cdot 07$, Fisher's exact test, two sided.

ANOVA of CMR perfusion slopes for all segments of the left ventricle versus MPS score (four points based on MPS classification of segments) had correlation $\mathrm{r}=0.64$ (SSFP) and $r=0.96$ (GRE-EPI).

\section{Reproducibility}

Intraclass correlation between two observers measuring myocardial wash-in slope showed fair agreement of consistency for the GRE-EPI sequence, $0 \cdot 86$ with CI $95 \% 0 \cdot 80-0 \cdot 90$. The SSFP sequence showed lower agreement, 0.53 CI 95\% 0.36$0 \cdot 66$.

\section{Discussion}

Previous studies have demonstrated that CMR perfusion imaging may be superior to MPS in the detection of myocardial ischaemia and significant coronary stenoses (Merkle et al., 2007; Schwitter et al., 2008, 2012) although CMR yet lacks the clinical prognostic documentation that is available for MPS. Still, there is no consensus regarding which sequence to prefer in CMR perfusion. SSFP was known to have higher SNR and CNR than spoiled gradient and echo-planar imaging (Wang et al., 2005; Gebker et al., 2007; Merkle et al., 2007) which gave hope for an advantage also in perfusion imaging. However, despite similarly high values of SNR and CNR also in this study, the sensitivity and specificity to detect abnormal myocardial perfusion was lower for SSFP compared with GREEPI. This was true for quantitative as well as clinical visual assessment. The effect of a T1-shortening agent such as gadolinium on different MR sequences is complex. Flip angle, echo time, repetition time and saturation recovery all interact in a complex manner, but it is likely that a GRE-EPI with a flip angle of $20^{\circ}$ confers a stronger T1 weighting than SSFP with a flip angle $50^{\circ}$, depending on the balance between T1 and T2 weighting in SSFP. Some of the superior SNR of SSFP may thus be produced by the combined T1/T2 weighting of balanced SSFP. Likewise, the high concentration of gadolinium contrast enhancement during first pass perfusion shortens both the $\mathrm{T} 1$ and the $\mathrm{T} 2 *$ relaxation, which perhaps is a disadvantage in a T1/T2-weighted sequence, where the increase in signal due to $\mathrm{T} 1 \mathrm{can}$ be reduced by the decrease in signal due to T2*.Furthermore, the appearance of artefacts may differ between sequences. SSFP is sensitive to 'dark rim artefact', DRA, which can be mistaken for a perfusion defect. It has been suggested that this artefact may be caused by Gibb's ringing (low resolution in the phase-encoding direction), by cardiac motion, magnetic susceptibility, or $\mathrm{T} 2 *$ effects due to the high concentration of cavity contrast during bolus injection (Di Bella et al., 2005; Gerber et al., 2008). However, there is no consensus as to when the DRA is due to artefact or a true reduction in perfusion (Di Bella et al., 2005). It has been suggested that a short-lived endocardial darkening favours artefact (Barkhausen et al., 2004). Other authors (Hautvast et al., 2011) have tried to circumvent this problem by investigating the perfusion gradient from many (60) sectors along the circumference of the left ventricle. Their method relies on a comparison between stress and rest that effectively nullifies the effect of the DRA, and on the fact that slow wash-in for the entire myocardial thickness increases the likelihood of the presence of a significant stenosis of the supply vessel. Still, significant difficulties remain for determining quantitative measures of CMR perfusion and the assessment of signal from scar areas (Gupta et al., 2012; Bratis and Nagel, 2013). Recently, Arai et al. showed that the highest area under curve for the detection of $>70 \%$ coronary stenosis on quantitative coronary angiography was obtained with a double bolus perfusion technique and absolute quantification of myocardial blood flow, compared with three different semiquantitative techniques, regardless of the presence of scar or not (Mordini et al., 2014). This technique needs to be evaluated in larger studies.

In studies where visual assessment is used, a higher perfusion contrast dose, $0 \cdot 075-0 \cdot 1 \mathrm{mmol} \mathrm{kg}^{-1}$, has been found to confer increased sensitivity and specificity of CMR perfusion detection of coronary stenoses defined with X-ray coronary angiography (Schwitter et al., 2008). This suggests that contrast dosing should be considered in light of the method of image assessment. While the human eye may be more sensitive to the difference in signal intensity caused by a high contrast dosing, available semiquantitative evaluation methods may not. In our hands, visual assessment was not inferior to quantitative measurements.

The field strength of the scanner is important for the selection of the sequence used for cardiac perfusion imaging, as at 3T, a T1-weighted EPI sequence has twice as high SNR as at 1.5 T (Gutberlet et al., 2006) and the SSFP sequence displays more susceptibility artefacts at $3 \mathrm{~T}$ compared with $1.5 \mathrm{~T}$ 


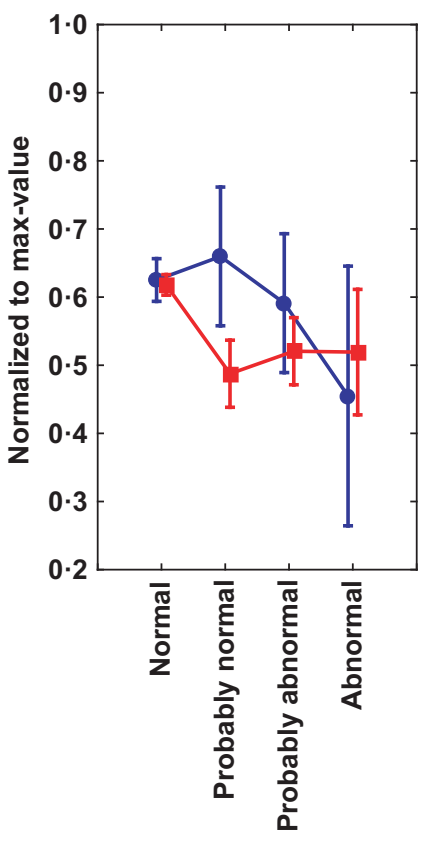

Basal

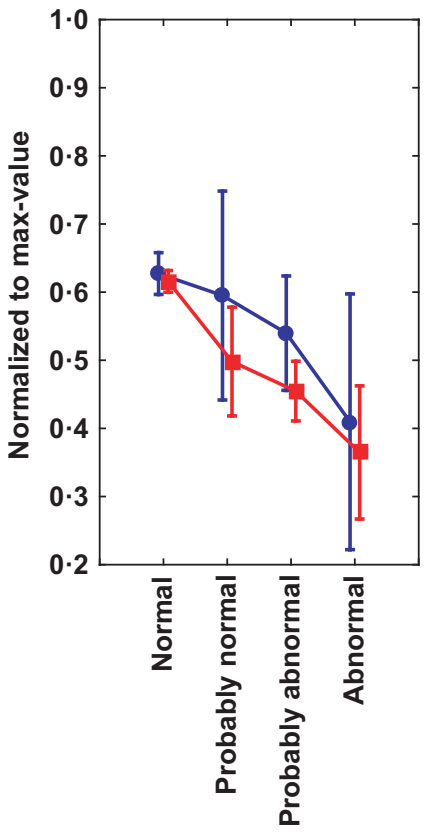

Basal

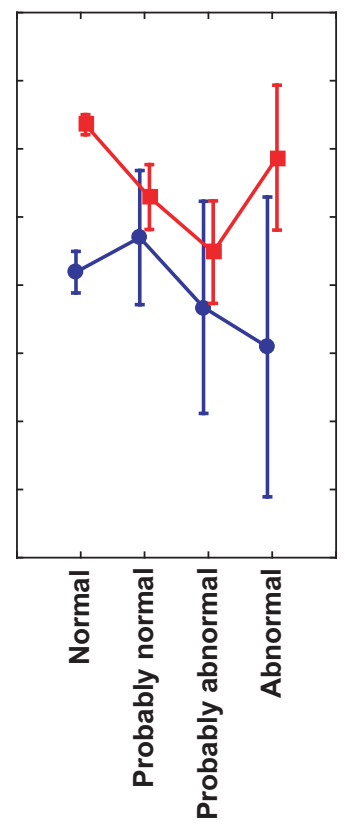

Mid

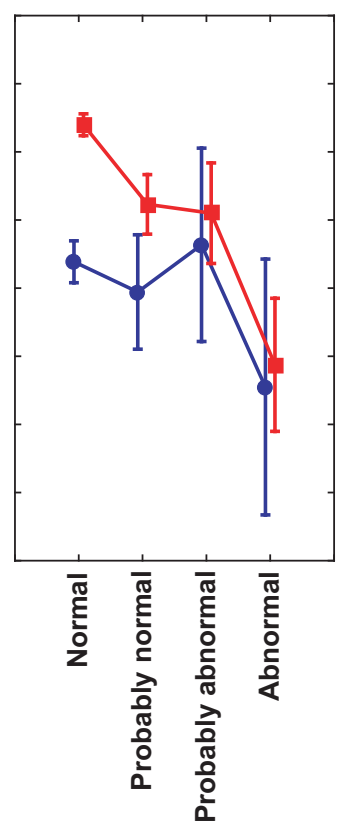

Mid

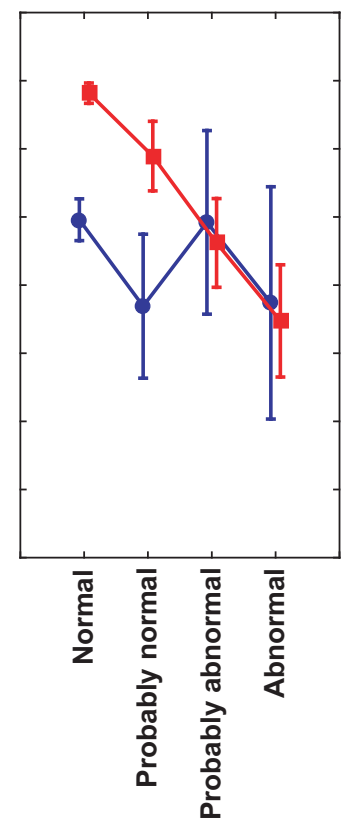

Apical

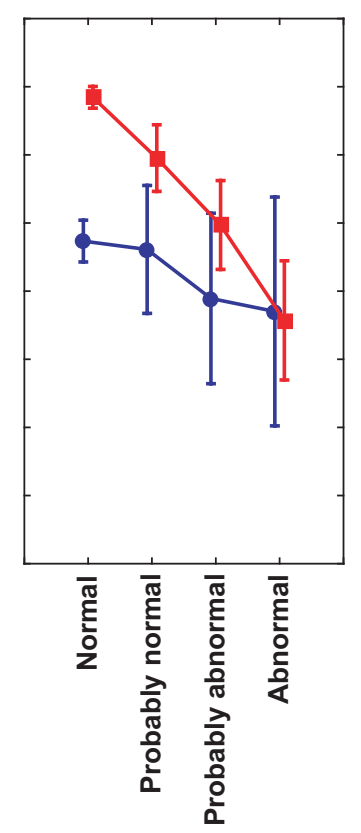

Apical

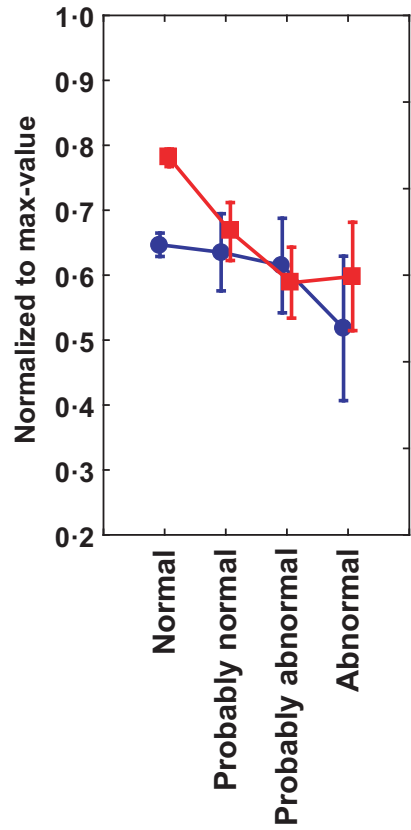

All regions

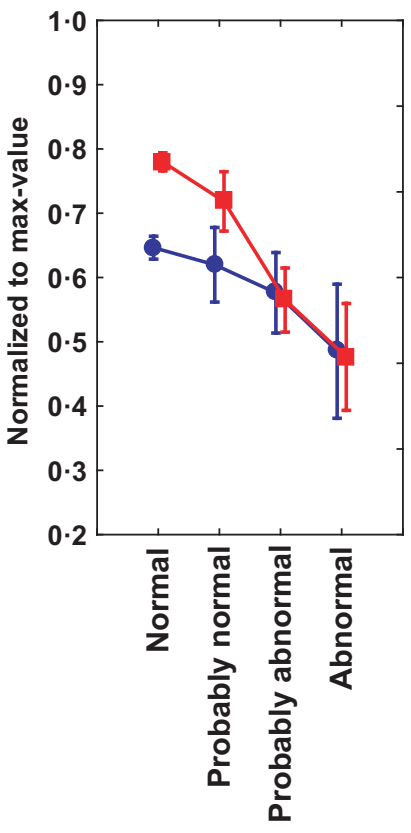

All regions

Figure 4 MPS scores (red line with squares) and MR slope (blue line with dots) during vasodilation. SSFP (upper panels) and GRE-EPI (lower panels). The three segmental levels base, middle and apex to the left and the aggregated results to the right. Values are normalized to maximum value in each individual to allow for comparisons. Normal, ischaemic and scar segments are all included.

(Gerber et al., 2008). This suggests that GRE-EPI might perform even better at $3 \mathrm{~T}$.

A number of limitations apply to this study as follows: the two CMR sequences were applied to two different cohorts, as the GRE-EPI sequence became available somewhat later. Even if both sequences had been available simultaneously, the appropriateness of performing two different stress tests on each patient would have been questionable. A head-to-head comparison of the two sequences was therefore not possible. MPS was selected for reference as it exploits the physiologic effect of a coronary stenosis which is the mechanism studied also in MR perfusion, but the sensitivity and specificity of MPS to detect coronary artery stenoses is imperfect even if it has recently been used to validate a 3D MRI perfusion 
sequence (Jogiya et al., 2014). Furthermore, as MPS has a dose-response (tracer signal versus flow) relationship that is partly determined by membrane function as well as coronary blood flow, this relationship has nonlinear components which can have added to some of the observed differences between the two perfusion sequences. Due to a limited availability of scanner time, patient recruitment was extended over a 3-year period.

In conclusion, this study shows significant differences between two CMR perfusion sequences as applied according to guidelines, with advantage to the GRE-EPI-based hybrid sequence despite lower SNR and CNR than the SSFP perfusion sequence. The GRE-EPI sequence produces images that closely follow the variation in MPS signal, suggesting that objective evaluation of myocardial perfusion by CMR may be within reach.

\section{Acknowledgments}

The technicians of the MRI and nuclear medicine units at Ryhov County Hospital, Jonkoping, are gratefully acknowledged for performing the patient studies. Siemens Healthcare gave access to the two perfusion sequences that at the time were work in progress, WIP. This study was supported by grants from the Medical Research Council of Southeast Sweden (Grant no 12437), Futurum, the County council of Jonkoping (Grants no 12440, 81851, 217261), Linkoping University, the County Council of Ostergotland (Grant no 281281) and the Swedish Heart-Lung Foundation (Grant no 20120449).

\section{Conflict of interest}

The authors have no conflict of interest.

\section{References}

Barkhausen J, Hunold P, Jochims $M$, et al. Imaging of myocardial perfusion with magnetic resonance. J Magn Reson Imaging (2004); 19: 750-757.

Bratis K, Nagel E. Variability in quantitative cardiac magnetic resonance perfusion analysis. J Thorac Dis (2013); 5: 357-359.

Carlsson M, Jogi J, Bloch KM, et al. Submaximal adenosine-induced coronary hyperaemia with $12 \mathrm{~h}$ caffeine abstinence: implications for clinical adenosine perfusion imaging tests. Clin Physiol Funct Imaging (2015); 35: 49-56.

Cerqueira MD, Weissman NJ, Dilsizian V, et al. Standardized myocardial segmentation and nomenclature for tomographic imaging of the heart. A statement for healthcare professionals from the Cardiac Imaging Committee of the Council on Clinical Cardiology of the American Heart Association. Int J Cardiovasc Imaging (2002); 18: 539-542.

Constantine G, Shan K, Flamm SD, et al. Role of MRI in clinical cardiology. Lancet (2004); 363: 2162-2171.

Di Bella EV, Parker DL, Sinusas AJ. On the dark rim artifact in dynamic contrastenhanced MRI myocardial perfusion studies. Magn Reson Med (2005); 54: 1295-1299.

Finn JP, Nael K, Deshpande V, et al. Cardiac MR imaging: state of the technology. Radiology (2006); 241: 338-354.

Fleischmann S, Koepfli P, Namdar M, et al. Gated (99 m)Tc-tetrofosmin SPECT for discriminating infarct from artifact in fixed myocardial perfusion defects. J Nucl Med (2004); 45: 754-759.

Gebker R, Schwitter J, Fleck E, et al. How we perform myocardial perfusion with cardiovascular magnetic resonance. J Cardiovasc Magn Reson (2007); 9: 539-547.
Gerber BL, Raman SV, Nayak K, et al. Myocardial first-pass perfusion cardiovascular magnetic resonance: history, theory, and current state of the art. J Cardiovasc Magn Reson (2008); 10: 18. doi:10.1186/1532-429X-10-18

Gibbons RJ, Balady GJ, Bricker JT. ACC/AHA 2002 guideline update for exercise testing: Summary article: a report of the American College of Cardiology American Heart Association task force on practice guidelines (Committee to update the 1997 exercise testing guidelines). J Am Coll Cardiol (2006); 48: 1731-1731.

Gould KL, Kirkeeide RL, Buchi M. Coronary flow reserve as a physiologic measure of stenosis severity. J Am Coll Cardiol (1990); 15: 459-474. Greenwood JP, Maredia N, Younger JF, et al. Cardiovascular magnetic resonance and single-photon emission computed tomography for diagnosis of coronary heart disease (CEMARC): a prospective trial. The Lancet (2012); 379: 453-460. doi: 10.1016/ S0140-6736(11)61335-4.

Griswold MA, Jakob PM, Heidemann RM, et al. Generalized autocalibrating partially parallel acquisitions (GRAPPA). Magn Reson Med (2002); 47: 1202-1210.

Gupta V, Kirisli HA, Hendriks EA, et al. Cardiac MR perfusion image processing techniques: a survey. Med Image Anal (2012); 16: 767-785

Gutberlet M, Noeske R, Schwinge K, et al. Comprehensive cardiac magnetic resonance imaging at 3.0 Tesla: feasibility and implications for clinical applications. Invest Radiol (2006); 41: 154-167.

Hautvast GL, Chiribiri A, Lockie T, et al. Quantitative analysis of transmural gradients in myocardial perfusion magnetic resonance images. Magn Reson Med (2011); 66: 1477-1487.
Hundley WG, Bluemke D, Bogaert JG, et al. Society for Cardiovascular Magnetic Resonance guidelines for reporting cardiovascular magnetic resonance examinations. J Cardiovasc Magn Reson (2009); 11: 5.

Jerosch-Herold M. Quantification of myocardial perfusion by cardiovascular magnetic resonance. J Cardiovasc Magn Reson (2010); 12: 57.

Jogiya R, Morton G, De Silva K, et al. Ischemic burden by Three-dimensional myocardial perfusion cardiovascular magnetic resonance: comparison with myocardial perfusion scintigraphy. Circ Cardiovasc Imaging (2014); 7: 647-654.

Kellman P, Arai AE. Imaging sequences for first pass perfusion - a review. J Cardiovasc Magn Reson (2007); 9: 525-537.

Kim HW, Farzaneh-Far A, Kim RJ. Cardiovascular magnetic resonance in patients with myocardial infarction: current and emerging applications. J Am Coll Cardiol (2009); 55: 116.

Kramer CM. When two tests are better than one: adding late gadolinium enhancement to first-pass perfusion cardiovascular magnetic resonance. J Am Coll Cardiol (2006); 47: 1639

Kramer CM, Barkhausen J, Flamm SD, et al. Standardized cardiovascular magnetic resonance imaging (CMR) protocols, society for cardiovascular magnetic resonance: board of trustees task force on standardized protocols. J Cardiovasc Magn Reson (2008); 10: 35.

Landis JR, Koch GG. The measurement of observer agreement for categorical data. Biometrics (1977); 33: 159-174.

Marcassa C, Bax JJ, Bengel F, et al. Med EESCWG, Nucl EA and Cardiovasc. Clinical value, cost-effectiveness, and safety of myo- 
cardial perfusion scintigraphy: a position statement. Eur Heart J (2008); 29: 557-563.

Merkle N, Wohrle J, Grebe O, et al. Assessment of myocardial perfusion for detection of coronary artery stenoses by steady-state, freeprecession magnetic resonance first-pass imaging. Heart (2007); 93: 1381-1385.

Mordini FE, Haddad T, Hsu LY, et al. Diagnostic accuracy of stress perfusion CMR in comparison with quantitative coronary angiography: fully quantitative, semiquantitative, and qualitative assessment. JACC Cardiovasc Imaging (2014); 7: 14-22.

Nandalur KR, Dwamena BA, Choudhri AF, et al. Diagnostic performance of stress cardiac magnetic resonance imaging in the detection of coronary artery disease: a meta-analysis. J Am Coll Cardiol (2007); 50: $1343-1353$.

Sakuma H. Magnetic resonance imaging for ischemic heart disease. J Magn Reson Imaging (2007); 26: 3-13.
Schwitter J, Wacker CM, van Rossum AC, et al. MR-IMPACT: comparison of perfusioncardiac magnetic resonance with singlephoton emission computed tomography for the detection of coronary artery disease in a multicentre, multivendor, randomized trial. Eur Heart J (2008); 29: 480-489.

Schwitter J, Wacker CM, Wilke N, et al. Superior diagnostic performance of perfusioncardiovascular magnetic resonance versus SPECT to detect coronary artery disease: the secondary endpoints of the multicenter multivendor MR-IMPACT II (Magnetic Resonance Imaging for Myocardial Perfusion Assessment in Coronary Artery Disease Trial). J Cardiovasc Magn Reson (2012); 14: 61.

Taylor AJ, Cerqueira M, Hodgson JM, et al. $\mathrm{ACCF} / \mathrm{SCCT} / \mathrm{ACR} / \mathrm{AHA} / \mathrm{ASE} / \mathrm{ASNC} / \mathrm{NASCI} /$ SCAI/SCMR 2010 Appropriate Use Criteria for Cardiac Computed Tomography. A Report of the American College of Cardiology Foundation Appropriate Use Criteria
Task Force, the Society of Cardiovascular Computed Tomography, the American College of Radiology, the American Heart Association, the American Society of Echocardiography, the American Society of Nuclear Cardiology, the North American Society for Cardiovascular Imaging, the Society for Cardiovascular Angiography and Interventions, and the Society for Cardiovascular Magnetic Resonance. J Cardiovasc Comput Tomogr (2010); 4: 407 e401-407 e433.

Wang Y, Moin K, Akinboboye O, et al. Myocardial first pass perfusion: steady-state free precession versus spoiled gradient echo and segmented echo planar imaging. Magn Reson Med (2005); 54: 1123-1129.

Zhang JJ, Raichlen JS, Kim SM, et al. Computer-assisted myocardial thickening analysis of gated MIBI SPECT images. Invest Radiol (1998); 33: 257-262. 